\title{
Application of verified neutron monitor yield function for GLE analysis
}

\author{
Alexander Mishev, ${ }^{a, b, *}$ Ilya Usoskin, ${ }^{a, b}$ Sergey Koldobskiy, ${ }^{b, c}$ Gennady Kovaltsov ${ }^{d}$ \\ and Leon Kocharov ${ }^{a}$ \\ ${ }^{a}$ Sodankylä Geophysical Observatory, \\ University of Oulu, Finland \\ ${ }^{b}$ Space Physics and Astronomy Research Unit, \\ University of Oulu, Finland \\ ${ }^{c}$ National Research Nuclear University MEPhI, \\ Moscow, Russia. \\ ${ }^{d}$ Ioffe Physical-Technical Institute, \\ St. Petersburg, Russia. \\ E-mail: alexander.mishev@oulu.fi, ilya.usoskin@oulu.fi
}

Systematic study of solar energetic particles provides an important basis to understand their acceleration and propagation in the interplanetary space. After solar eruptive processes, such as solar flares and/or coronal mass ejections, solar ions are accelerated to high energy. In the majority of cases, the maximum energy of the accelerated solar ions is several tens of $\mathrm{MeV} /$ nucleon, but in some cases, it exceeds $100 \mathrm{MeV} /$ nucleon or even reaches the $\mathrm{GeV} /$ nucleon range. In this case, the energy is high enough, so that solar ions generate an atmospheric cascade in the Earth's atmosphere, whose secondary particles reach the ground, being eventually registered by ground-based detectors, specifically neutron monitors. This particular class of events is known as ground-level enhancements (GLEs). Several methods for analysis of GLEs, using neutron monitor data were developed over the years. Here, we present a method for assessment of the spectral and angular characteristics of the GLEs using data from the world-wide NM network, namely by modeling the global neutron monitor network response with a new verified yield function. The method is based on consecutive steps, specifically detailed computation of asymptotic cones and rigidity cut-off of each station used in the analysis and optimization of the global neutron monitor response over experimental and modeled count rate increases. The method is compared with other methods, including in-situ measurements. A very good agreement between our method and space-borne measurements with PAMELA space probe, specifically the derived fluence of solar protons during GLE 71 was achieved, therefore verification of the method is performed.

$3^{\text {th }}$ International Cosmic Ray Conference (ICRC 2021)

July 12th-23rd, 2021

Online - Berlin, Germany

\footnotetext{
${ }^{*}$ Presenter
} 


\section{Introduction}

A specific class of solar energetic particles (SEP) events, that can be observed at the ground level by registration of the sub-products of induced atmospheric showers, called ground-level enhancements (GLEs) [1,2], invokes specific interest, giving a basis to understand the possible acceleration scenarios as well as the interplanetary transport [3-5].

As a result of solar eruptive processes, viz. solar flares, and/or coronal mass ejection (CMEs), solar ions can be accelerated to high energies, i.e. producing SEPs [6, 7]. They penetrate into the Earth's atmosphere and if their energy is about GeV/nucleon or greater, produce nuclearelectromagnetic-meson shower of secondaries, that can be registered by ground-based detectors, specifically neutron monitors (NMs) [8, 9]. Here, we performed a revised analysis of 17 May 2017 GLE \# 71, using newly computed and verified NM yield function for several altitudes and compared the derived fluence with direct measurements e.g. by the PAMELA space probe.

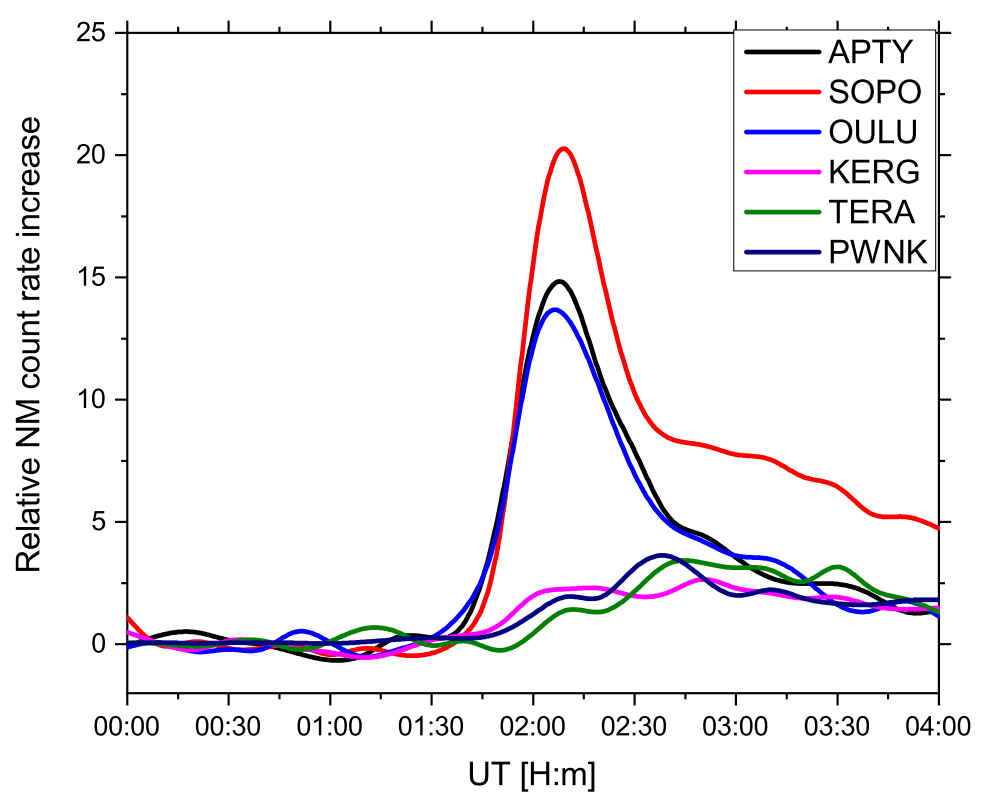

Figure 1: Observed NM count rate increases of selected stations during GLE \# 71 on 17 May 2012.Data available at gle.oulu.fi.

\section{Method for GLEs analysis using NM data}

Methods for analysis of GLEs using NM data have been developed over the years. They are based on modeling of the global NM network response and unfolding $n$ model parameters over the experimental records [1, 10-12]. Here we employed method whose description and application is given elsewhere [13-16]. In summary, the method involves consecutive steps of detailed computation of asymptotic cones and rigidity cut-offs of each NM station whose records 
are used in the analysis, modelling and corresponding optimization of the global NM response over experimental and modeled count rate increase. In this study we assumed a modified power law with variable slope rigidity spectrum of SEPs similarly to [10]:

$$
J_{\|}(P)=J_{0} P^{-(\gamma+\delta \gamma(P-1))}
$$

where $J_{\|}(P)$ is the particle flux, with given rigidity $P$, arriving from the Sun along the axis of symmetry whose direction is defined by geographic coordinate angles $\Psi$ and $\Lambda$ (latitude and longitude), $\gamma$ is the power-law spectral slope at rigidity $\mathrm{P}=1 \mathrm{GV}, \delta \gamma$ is the rate of the spectrum steepening.

The pitch angle distribution (PAD) is assumed to be a superposition of two Gaussian like distributions, which allows us to model a complicated (superposition of Gaussians), including bidirectional, particle flow:

$$
G(\alpha(P)) \propto \exp \left(-\alpha^{2} / \sigma_{1}^{2}\right)+B * \exp \left(-(\alpha-\pi)^{2} / \sigma_{2}^{2}\right)
$$

where $\alpha$ is the pitch angle, $\sigma_{1}$ and $\sigma_{2}$ are parameters describing the width of the pitch angle distribution, $B$ is a parameter corresponding to the contribution of the particle flux arriving from the anti-sun direction. Here, the modeling of the global network NM response was performed with newly computed and validated by experimental data NM yield function, [17-19], while the unfolding was performed over the set of $n$ model parameters by minimizing the difference between the modeled and measured NM responses, that is by an inverse problem solution [20,21].

As first step, we computed the asymptotic trajectories and cut-off rigidities of NMs of all stations considered in the analysis, using MAGNETOCOSMICS code [22] employing a combination of IGRF model as internal field and the Tsyganenko 89 model [23] as the external field, respectively. This combination of magnetospheric models provides straightforward and precise computation [24]. As example we present the asymptotic directions of selected NMs during GLE \# 71 on 17 May 2012 (Fig.2).

\section{Analysis of \# GLE 71 using NM data}

GLE \# 71 occurred on 17 May 2012, following eruptions in the active region NOAA 11476, which produced a CME and a M5.1 flare at 01:25 UT. The active region was on the west side at N07 W88, so that the Earth was well magnetically connected to the eruption core. On the ground, the global NM network recorded moderate enhancement of the count rates of several stations around 01:50 UT, with slightly greater signals observed in APTY, OULU, and SOPO/SOPB NMs (Fig.1). We note, that the bulk of NMs registered only marginal count rate increases, which implied a large anisotropy of the arriving SEPs, specifically during the event onset. The SEP flux was observed above the background of GCRs for about two and a half hours.

Using de-trended NM records [25] retrieved from the GLE database (for details see the http://gle.oulu.fi), we derived the spectral and angular characteristics of SEPs (Fig.3). In contrast to the previous studies, here we expanded considerably the time interval and the accuracy of the derived information [26]. 


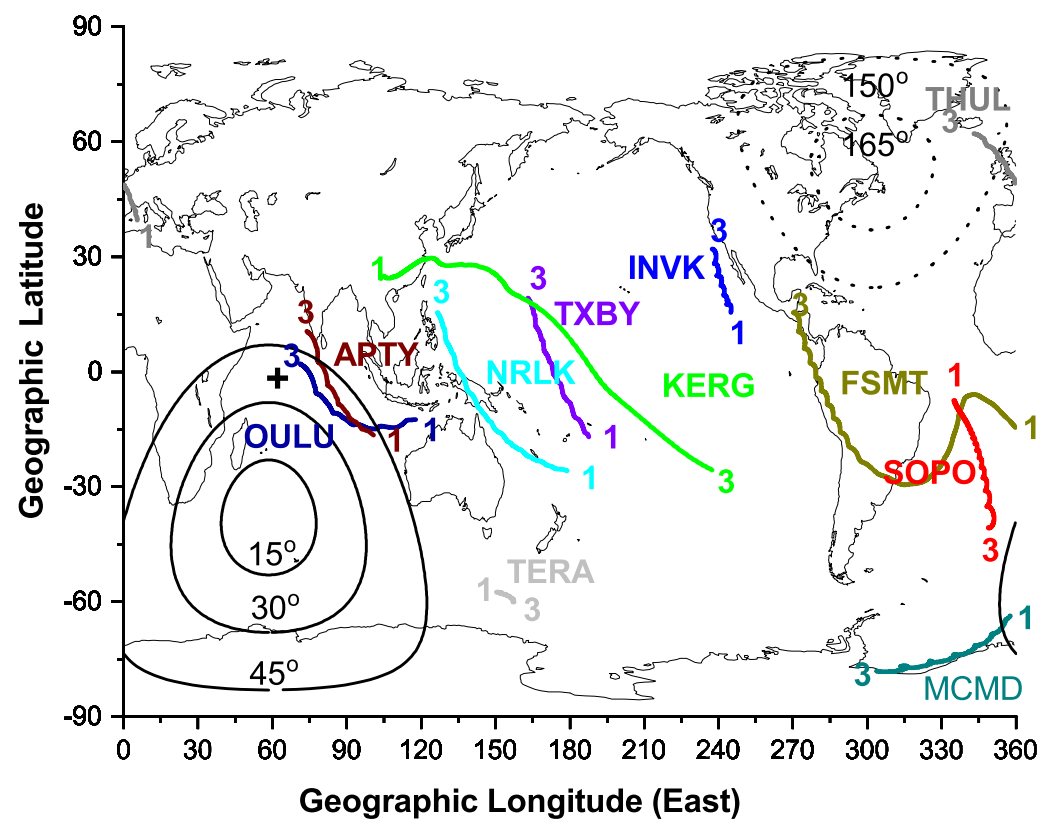

Figure 2: Asymptotic directions of selected NMs during GLE \# 71 on 17 May 2021. The color lines and acronyms and numbers depict the asymptotic directions and rigidities, which are plotted in the rigidity range $1-5 \mathrm{GV}$. The lines of equal pitch angles relative to the anisotropy axis are plotted for $15^{\circ}, 30^{\circ}$ and $45^{\circ}$ for sunward direction (solid lines), $165^{\circ}, 150^{\circ}$ for the anti-Sun direction (dashed lines), respectively. The cross depicts the measured by ACE space-probe IMF direction.

We distinguished three phases of the event: initial (01:50-02:25 UT), when a relatively hard spectrum, a constant increase of SEP flux and complicated PAD were derived; main phase (02:2503:05 UT), when a steady softening of the SEP spectra and decrease of the SEP flux were derived, accordingly a tendency of decrease of the steepening $\delta \gamma$ of the spectrum was observed, and late phase of the event (after 03:05 UT), when a pure power-law SEP spectrum and wider a nearly isotropic PAD were revealed.

Here, we note that the approach of initial (strong anisotropy and modified power-law or exponential spectrum), main (moderate anisotropy, maximal particle flux and modified power-law spectrum) and late (isotropic flux and pure power-law spectrum) phases of the event is in agreement with that of the widely discussed prompt and delayed components [11]. The initial and early main phases of the event correspond roughly to the prompt component, whilst the late phase corresponds to the delayed component.

Here we computed the event integrated particle fluence and very good agreement with the direct measurements made by PAMELA space-probe was achieved [27] (Fig.4), the details are given in [28]. We note, that PAMELA allowed one to reveal SEPs over GCR background for rigidities less than about $2 \mathrm{GV}$. Therefore, the spectra and PAD in the higher-energy range were retrieved using solely the global NM network. Besides, good agreement with SOHO/EPHIN measurements fitted with pure power-law, specifically in the energy range 300-700 MeV/nucleon, was also seen [29]. 

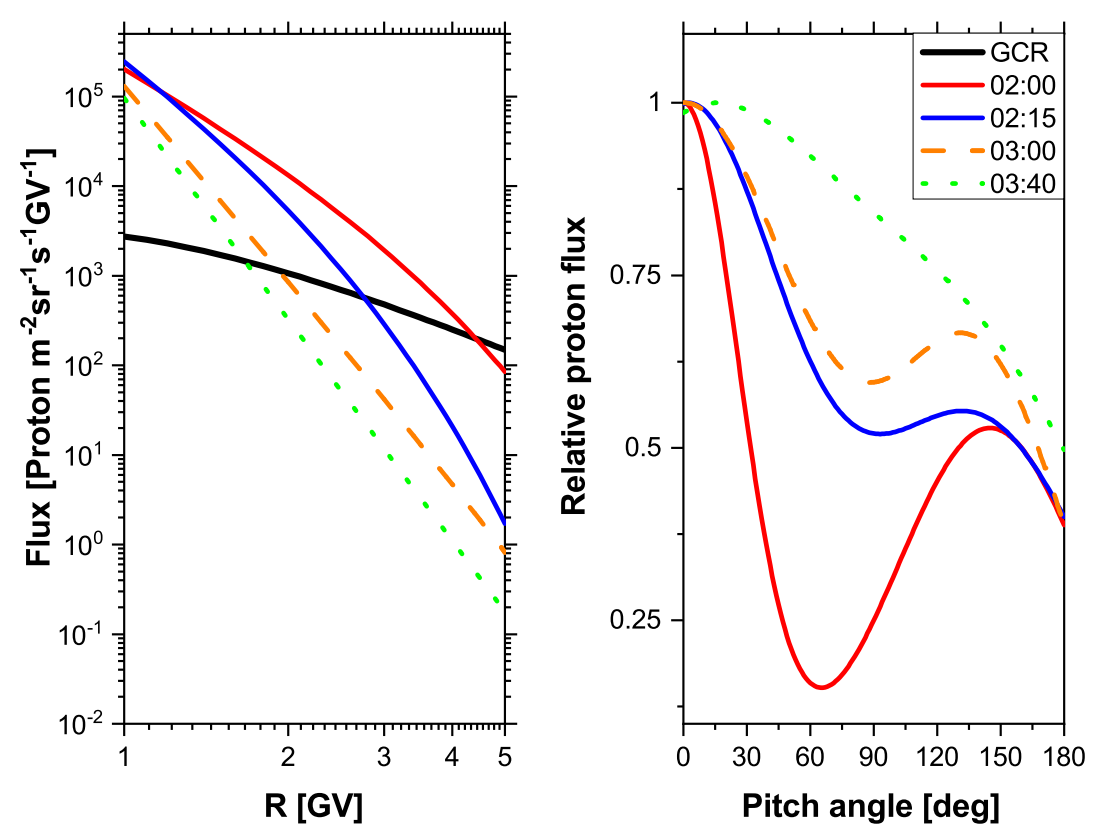

Figure 3: Derived spectra and PAD during selected stages of GLE \# 71.

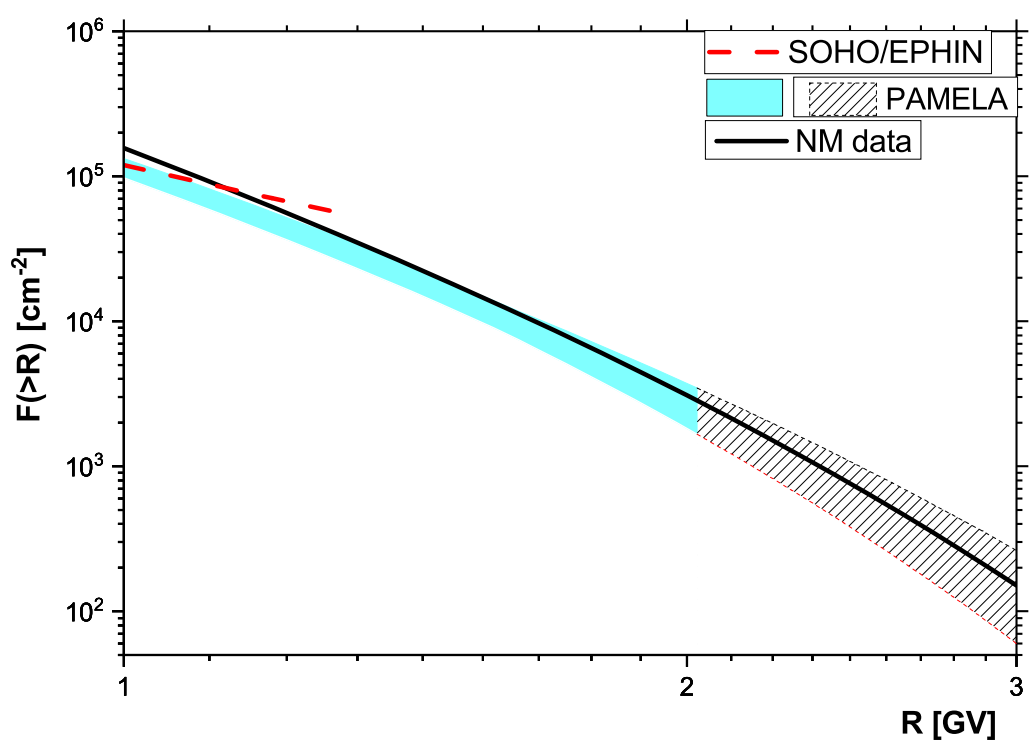

Figure 4: SEP fluence during GLE \# 71 computed using NM, PAMELA, and SOHO/EPHIN data reconstructions as denoted in the legend. The light-blue filled and hatched area correspond to the PAMELA data up to $2 \mathrm{GV}$ and the corresponding data extrapolation above $2 \mathrm{GV}$ respectively. The direct measurements of PAMELA are parameterized using Ellison-Ramaty spectral shape where the $1 \sigma$ uncertainties are shown with the filled area. The direct measurements of SOHO/EPHIN are parameterized using power-law spectrum. 
Most important was a very similar complicated angular distribution of the SEPs that was revealed by direct PAMELA observations [30]. Therefore, we can claim that our method of analysis using a verified NM yield function corresponding to the specific altitude of the station a.s.l. within robust and reliable optimization is validated with good agreement by direct measurements [31].

\section{Conclusions}

In this study, we derived the rigidity spectrum and PAD of high-energy SEPs in the course of GLE \# 71 on 17 May 2012. The analysis was performed employing a robust optimization method, using data from the global NM network, newly computed and verified NM yield function. The accuracy of the derived spectra and PAD was greatly improved comparing to our previous studies. A very good agreement of the derived SEP characteristics, specifically fluence and PAD with direct measurements by the PAMELA space-borne detector was achieved. Therefore, this specific method for data analysis of strong SEP events using NM records is verified at least for a single event and gives a good basis for further quantification of solar proton acceleration [32, 33].

\section{Acknowledgements}

This work was supported by the Academy of Finland (project 330064 QUASARE, 330427 HEAIM 2 and 321882 ESPERA). The work benefited from discussions in the framework of the International Space Science Institute International Team 441: High EneRgy sOlar partICle Events Analysis (HEROIC). We acknowledge all the PIs and colleagues from the neutron monitor stations, who kindly provided the data used in this analysis.

\section{References}

[1] M. Shea and D. Smart, Possible evidence for a rigidity-dependent release of relativistic protons from the solar corona, Space Science Reviews 32 (1982), 251-271.

[2] S. Poluianov, I. Usoskin, A. Mishev, A. Shea, and D. Smart, GLE and sub-GLE redefinition in the light of high-altitude polar neutron monitors, Solar Physics 292 (2017), no. 11176.

[3] H. Debrunner, E. Flückiger, H. Gradel, J. Lockwood, and R. McGuire, Observations related to the acceleration, injection, and interplanetary propagation of energetic protons during the solar cosmic ray event on February 16, 1984, Journal of Geophysical Research 93 (1988), no. A7 7206-7216.

[4] D. Reames, Particle acceleration at the sun and in the heliosphere, Space Science Reviews 90 (1999), 413-491.

[5] L. Kocharov, S. Pohjolainen, A. Mishev et al., Investigating the origins of two extreme solar particle events: Proton source profile and associated electromagnetic emissions, The Astrophysical Journal 839 (2017), no. 279.

[6] M. Desai and J. Giacalone, Large gradual solar energetic particle events, Living Reviews in Solar Physics 13 (2016), no. 13. 
[7] K.-L. Klein and S. Dalla, Acceleration and propagation of solar energetic particles, Space Science Reviews 212 (2017), no. 3-4 1107-1136.

[8] J. Simpson. The Cosmic Ray Nucleonic Component: The Invention and Scientific Uses of the Neutron Monitor, Space Science Reviews 93 (2000), 11-32.

[9] M. Aschwanden, GeV particle acceleration in solar flares and ground level enhancement (GLE) events, Space Science Reviews 171 (2012), no. 1-4 3-21.

[10] J. Cramp, M. Duldig, E. Flückiger, J. Humble, M. Shea and D. Smart, The October 22, 1989, solar cosmic enhancement: ray an analysis the anisotropy spectral characteristics, Journal of Geophysical Research 102 (1997), no. A11 24 237-24 248.

[11] E. Vashenyuk, Y. Balabin, J. Perez-Peraza, A. Gallegos-Cruz and L. Miroshnichenko, Some features of the sources of relativistic particles at the sun in the solar cycles 21-23, Advances Space Research 38 (2006), no. 3 411-417.

[12] A. Mishev and I. Usoskin, Analysis of the ground level enhancements on 14 July 2000 and on 13 December 2006 using neutron monitor data, Solar Physics 291 (2016), no. 4 1225-1239.

[13] A. Mishev, S. Poluianov and I. Usoskin, Assessment of spectral and angular characteristics of sub-GLE events using the global neutron monitor network, Journal of Space Weather and Space Climate 7 (2017) A28.

[14] A. Mishev, I. Usoskin, O. Raukunen, M. Paassilta, E. Valtonen, L. Kocharov and R. Vainio, First analysis of GLE 72 event on 10 September 2017: Spectral and anisotropy characteristics, Solar Physics 293 (2018) 136.

[15] A. Mishev and I. Usoskin, Assessment of the radiation environment at commercial jet-flight altitudes during GLE 72 on 10 September 2017 using neutron monitor data, Space Weather 16 (2018), no. 12 1921-1929.

[16] A. Mishev, S. Koldobskiy, L. Kocharov and I. Usoskin, GLE \# 67 Event on 2 November 2003: An Analysis of the Spectral and Anisotropy Characteristics Using Verified Yield Function and Detrended Neutron Monitor Data, Solar Physics 296 (2021), no. 579.

[17] A. Mishev , I. Usoskin, and G. Kovaltsov. Neutron Monitor Yield Function: New Improved computations. J. Geophys. Res. (Space Phys.), 118, (2013), 2783-2788.

[18] S.A. Koldobskiy, , V. Bindi, C. Corti, G. A. Kovaltsov, and I. G. Usoskin. Validation of the Neutron Monitor Yield Function Using Data from AMS-02 Experiment 2011-2017. J. Geophys. Res. (Space Phys.), 124, (2019) 2367-2379

[19] A.L. Mishev, S.A. Koldobskiy, G.A. Kovaltsov, A. Gil, and I.G. Usoskin. Updated NeutronMonitor Yield Function: Bridging Between In Situ and Ground-Based Cosmic Ray Measurements. J. Geophys. Res. (Space Phys.), 125 (2020), e2019JA027, 433.

[20] A. Tikhonov, A. Goncharsky, V. Stepanov and A. Yagola, Numerical Methods for Solving ill-Posed Problems. Kluwer Academic Publishers, Dordrecht, 1995. 
[21] S. Mavrodiev, A. Mishev and J. Stamenov, A method for energy estimation and mass composition determination of primary cosmic rays at the Chacaltaya observation level based on the atmospheric Cherenkov light technique, Nucl. Instr. and Methods in Phys. Res. A 530 (2004), no. 3 359-366.

[22] L. Desorgher, E. Flückiger, M. Gurtner, M. Moser, and R. Bütikofer, A GEANT 4 code for computing the interaction of cosmic rays with the earth's atmosphere, International Journal of Modern Physics A 20 (2005), no. A11 6802-6804.

[23] N. Tsyganenko, A magnetospheric magnetic field model with a warped tail current sheet, Planetary and Space Science 37 (1989), no. 15-20.

[24] J. Nevalainen, I. Usoskin, and A. Mishev, Eccentric dipole approximation of the geomagnetic field: Application to cosmic ray computations, Advances in Space Research 52 (2013), no. 1 22-29.

[25] I. Usoskin, S. Koldobskiy, G. Kovaltsov et al., Revised GLE database: Fluences of solar energetic particles as measured by the neutron-monitor network since 1956, Astronomy and Astrophysics, 640 (2020), A17.

[26] A. Mishev, L. Kocharov, and I. Usoskin, Analysis of the ground level enhancement on 17 May 2012 using data from the global neutron monitor network, Journal of Geophysical Research 119 (2014) 670-679.

[27] A. Bruno, A., G. Bazilevskaya, M. Boezio et al., Solar energetic particle events observed by the PAMELA mission, Astrophysical Journal, 862 (2018), no. 2, 97.

[28] S. Koldobskiy, G. A. Kovaltsov, A. Mishev, and I. G. Usoskin, New Method of Assessment of the Integral Fluence of Solar Energetic (>1 GV Rigidity) Particles from Neutron Monitor Data, Solar Physics, 294, (2019), 94.

[29] P. Kühl, , S. Banjac, N. Dresing, R. Gomez-Herrero, B. Heber, A. Klassen, and C. Terasa, Proton intensity spectra during the solar energetic particle events of May 17, 2012 and January 6, 2014,Astronomy and Astrophysics, 576, (2015), A120.

[30] O. Adriani, G. Barbarino, G. Bazilevskaya et al., PAMELA's measurements of magnetospheric effects on high-energy solar particles, Astrophysical Journal Letters, 801, (2015), no. 1, L3.

[31] A. Mishev, S. Koldobskiy, I. Usoskin, L. Kocharov and G. A. Kovaltsov, Application of the verified neutron monitor yield function for an extended analysis of the GLE \# 71 on 17 May 2012, Space Weather 19 (2021), e2020SW002626.

[32] L. Kocharov, S. Pohjolainen, M.J. Reiner, A. Mishev, H. Wang, I. Usoskin and R. Vainio, Spatial Organization of Seven Extreme Solar Energetic Particle Events, Astrophysical Journal Letters 862 (2018), no. 2 L20.

[33] L. Kocharov, M. Pesce-Rollins, T. Latinen, A. Mishev et al., , Interplanetary Protons versus Interacting Protons in the 2017 September 10 Solar Eruptive Event, The Astrophysical Journal 890 (2020), 13. 